\title{
Health State Utilities of Patients With Hepatitis B and $C$ and Hepatitis-related Conditions in Japan
}

Hiroki Sugimori ( $\nabla$ hsugimor@ic.daito.ac.jp )

Daito Bunka University

Maki Hirao

Saiseikai Central Hospital

Ataru Igarashi

Yokohama City University

Hiroshi Yatsuhashi

National Hospital Organization Nagasaki Medical Center

Shunya Ikeda

International University of Health and Welfare

Naohiko Masaki

National Sanatorium Tamazenshoen

Hiroshi Yotsuyanagi

Tokyo University

Takeshi Yoda

Kawasaki Medical School

Takeshi Odajima

Japanese Red Cross Kanto-Koshinetsu Block Blood Center

Tomoyuki Takura

Tokyo University

Tomohiro Hirao

Kagawa University

\section{Research Article}

Keywords: hepatitis, health state utilities, quality of life, EQ-5D-5L, the cost-utility analysis

Posted Date: January 24th, 2022

DOI: https://doi.org/10.21203/rs.3.rs-1264240/v1

License: (9) This work is licensed under a Creative Commons Attribution 4.0 International License.

Read Full License 

Health state utilities of patients with hepatitis B and C and hepatitis-related conditions in Japan

Hiroki Sugimori ${ }^{1 \&}$, Maki Hirao ${ }^{2, \# b}$, Ataru Igarashi ${ }^{3}$, Hiroshi Yatsuhashi ${ }^{4}$, Shunya Ikeda $^{5}$, Naohiko Masaki ${ }^{6}$, Hiroshi Yotsuyanagi ${ }^{7}$, Takeshi Yoda ${ }^{8}$, Takeshi Odajima ${ }^{9}$, Tomoyuki Takura ${ }^{10}$, Tomohiro Hirao ${ }^{11}$

$<$ ORCID Number $>$

Hiroki Sugimori; 0000-0003-1234-417X

Maki Hirao; 0000-0001-7148-9278

Ataru Igarashi; 0000-0001-6307-6916

Shunya Ikeda; 0000-0001-6180-246X

Hiroshi Yatsuhashi; 0000-0001-5487-3403

Naohiko Masaki; 0000-0002-0631-8648

Hiroshi Yotsuyanagi; 0000-0001-7882-5262

Takeshi Yoda; 0000-0001-6950-5056

Takeshi Odajima; not available

Tomoyuki Takura; 0000-0002-5987-9254

Tomohiro Hirao; 0000-0002-9824-565X

${ }^{1}$ Department of Nursing, Faculty of Sports and Health Science, Daito Bunka University, Saitama, Japan

${ }^{2}$ Department of Hematology, Saiseikai Central Hospital, Tokyo, Japan

${ }^{3}$ Unit of Public Health and Preventive Medicine, School of Medicine Medical Course, Yokohama City University, Kanagawa, Japan

${ }^{4}$ National Hospital Organization (NHO) Nagasaki Medical Center, Nagasaki, Japan

${ }^{5}$ School of Medicine, International University of Health and Welfare, Chiba, Japan

${ }^{6}$ National Sanatorium Tamazenshoen, Tokyo, Japan

${ }^{7}$ Division of Infectious Diseases, The Institute of Medical Science, The University of Tokyo, Tokyo, Japan

${ }^{8}$ Department of Public Health, Kawasaki Medical School, Okayama, Japan

${ }^{9}$ Japanese Red Cross Kanto-Koshinetsu Block Blood Center, Tokyo, Japan 
${ }^{10}$ Department of Healthcare Economics and Health Policy, Graduate School of Medicine, The University of Tokyo, Tokyo, Japan

${ }^{11}$ Department of Public Health, Faculty of Medicine, Kagawa University, Kagawa, Japan

${ }^{\# a}$ Current Address: 560 Iwadono, Higashimatsuyama City, Saitama, Japan

${ }^{\# b}$ Current Address: 1-4-17 Mita, Minato-ku, Tokyo, Japan

Corresponding author

Hiroki SUGIMORI, MD PhD MMedSc

- Daito Bunka University.

- Depertment of Nursing, Faculty of Sports and Health Science, Chairperson, Professor

- Graduate School of Sports and Health Science, Dept. of Preventive. Medicine., Professor

- 560 Iwadono, Higashimatsuyama City, Saitama, 3558501, Japan

- Tel: +81-493-31-1503 (Ext. 6190), Fax: +81-493-31-1528

•E-mail: hsugimor@ic.daito.ac.jp 


\section{Abstract}

Objective: Health state utilities are global measurements of quality of life and have been used to evaluate health outcomes for the cost-utility analysis. This study aimed to estimate the health state utilities of patients with hepatitis B (HB), hepatitis C (HC), and hepatitis-related diseases in Japan.

Materials and methods: We distributed a self-administered questionnaire, including the EuroQol 5Dimension 5-Level (EQ-5D-5L), to 9,952 outpatients with several clinical conditions caused by HB or HC virus infection (such as asymptomatic chronic hepatitis, chronic hepatitis, compensated cirrhosis, and decompensated cirrhosis) and estimated the condition-specific utilities of patients with HB or HC. In patients with more severe conditions (patients with acute hepatitis, fulminant hepatitis, and hepatocellular carcinoma and patients undergoing post-liver transplantation), the utilities of these severe conditions were estimated by three hepatitis experts using the EQ-5D-5L.

Results: The means of the utilities for acute hepatitis, fulminant hepatitis, asymptomatic chronic hepatitis, chronic hepatitis, compensated cirrhosis, compensated cirrhosis, hepatocellular carcinoma stage I/II, hepatocellular carcinoma stage III/IV, and post-liver transplantation were $0.529,-0.111,0.904,0.868$, $0.845,0.722,0,675,0,428$, and 0.651 and $0.876,0.821,0.737,0.671,0.675,0.428$, and 0.651 in HB and $\mathrm{HC}$, respectively.

Conclusion: To the best of our knowledge, this is the first study that comprehensively assessed the health state utilities of patients with $\mathrm{HB}, \mathrm{HC}$ and hepatitis-related conditions from a nationwide survey in Japan using the EQ-5D-5L.

Keywords: hepatitis, health state utilities, quality of life, EQ-5D-5L, the cost-utility analysis 


\section{Introduction}

Viral hepatitis is the most common blood-borne infection worldwide, causing hepatitis, hepatic cirrhosis, and hepatocellular carcinoma (HCC) [1-3]. In Japan, approximately 3\% of people are infected with hepatitis $\mathrm{C}$ virus (HCV) infection, resulting in more than 30,000 liver-related deaths annually [4-6]. Although most patients with chronic hepatitis $(\mathrm{CH})$ are asymptomatic in the early phases of the disease $[7,8]$, the disease can steadily progress to crucial symptomatic life-threatening liver conditions such as cirrhosis and liver cancer [9-12].

Guidelines for vaccination, viral testing, and treatment of hepatitis B (HB) and hepatitis C (HC) have been developed [7-9]. Moreover, several studies have reported on health-related quality of life (HRQoL) in patients with HB and HC [10-11]. However, in Japan, the assessment of hepatitis treatment, such as the Health Technology Assessment (HTA), could not provide sufficient information on the utilities of each HB- and HC-related condition and economic outcomes of their treatments. It has been suggested that each country should develop its own utilities because some studies have found that there are differences between countries in the observed quality weights for cultural, racial, and ethnic reasons [12].

A quality-adjusted life year (QALY) is a measure of disease burden, calculated as follows: 1 year of life is multiplied by a utility factor, where a utility of 0 represents death and a utility of 1 represents perfect health. In principle, the utility can be negative when the quality of life is judged to be worse than being dead. Using QALYs, we assume that health or health improvement can be measured based on the amount of time spent in various health conditions and make a decision on treatment. Thus, QALY is necessary for a cost-effectiveness analysis [13].

There are many methods for calculating the utilities. The National Institute for Health and Care Excellence (NICE) guidelines in the UK, which produce evidence-based guidelines for health, public health, and social care practitioners, recommend that the EuroQol 5-Dimension (EQ-5D) is the preferred measure of HRQoL in adults. The EQ-5D is universally used and has been validated in many populations, and it is composed of five dimensions of health: mobility, self-care ability, ability to undertake usual activities, pain and discomfort, and anxiety and depression. The system has been designed in such a way that people can describe their own HRQoL [14]. However, there are few or no such studies using a new descriptive system, EuroQol 5-Dimension 5-Level (EQ-5D-5L), with five response levels (no problems, slight problems, moderate problems, severe problems, and extreme problems) in patients with hepatitis in Japan.

Our study aimed to estimate the health state utilities of HB, HC, and hepatitis-related liver diseases in Japan, separately, using the Japanese version of the EQ-5D-5L [15]. The utilities of asymptomatic chronic hepatitis ( $\mathrm{ACH}$ ), $\mathrm{CH}$, compensated cirrhosis (CC), and decompensated cirrhosis (DC) caused by $\mathrm{HB}$ virus or $\mathrm{HC}$ virus infection were obtained from outpatients. However, since it is difficult for patients with severe liver conditions, such as HCC, acute hepatitis (AH), and fulminant 
hepatitis (FH), and patients undergoing post-liver transplantation (PLT) to evaluate their own conditions, in this study, we estimated the utility of these conditions from hepatitis experts. 


\section{Methods}

\section{Subjects}

From February 1 to July 31, 2012, we conducted a mail survey and distributed a self-administered questionnaire to 9,952 outpatients with $\mathrm{CH}$, hepatic cirrhosis, or $\mathrm{HCC}$ caused by hepatitis B virus (HBV) or HCV infection at 34 medical facilities, including the National Hospital Organization and National Center for Global Health and Medicine in Japan. The collection rate was 63.6\%, and 6,331 eligible subjects were included in our study. The survey comprised basic demographic information such as sex, age, disease history, and EQ-5D-5L score.

Patients selected their own disease in the following six medical conditions: (1) $\mathrm{CH},(2)$ hepatic cirrhosis, (3) HCC, (4) viral carrier, (5) fatty liver, and (6) others. Patients with hepatic cirrhosis who had at least one of the following conditions were diagnosed with DC: ascitic fluid, encephalosis, or albumin level $\leq 3.0 \mathrm{~g} / \mathrm{dL}$. None of the patients was diagnosed with CC. Patients who were viral carriers were diagnosed with $\mathrm{ACH}$. When patients selected more than one disease, the diseases were redefined, as shown in Table 1. Patients with HCC and HBV and HCV infections were excluded from this study.

Table 1. Disease classification of hepatitis-related conditions.

\begin{tabular}{|l|l|}
\hline Redefined condition & Conditions selected by patients \\
\hline Chronic hepatitis & $(1)(4),(1)(5),(1)(4)(5)$ \\
\hline Hepatic cirrhosis* & $(1)(2),(2)(4),(2)(5),(1)(2)(4),(1)(2)(5),(1)(2)(4)(5)$ \\
\hline Hepatocellular carcinoma & $\begin{array}{l}(1)(3),(2)(3),(3)(4),(3)(5),(1)(2)(3),(1)(3)(4),(2)(3)(4),(2)(3)(5), \\
(1)(3)(4)(5),(1)(2)(3)(5)\end{array}$ \\
\hline Asymptomatic hepatitis & $(4)(5)$ \\
\hline Others & $(1)(6),(4)(6),(5)(6),(1)(3)(6),(1)(4)(6)$ \\
\hline
\end{tabular}

(1) chronic hepatitis, (2) hepatic cirrhosis, (3) hepatocellular carcinoma, (4) viral carrier, (5) fatty liver, and (6) others. *Patients who had at least one of the following conditions were diagnosed with decompensated cirrhosis: ascitic fluid, encephalosis, or albumin level $\leq 3.0 \mathrm{~g} / \mathrm{dL}$. Others were diagnosed with compensated cirrhosis.

\section{EuroQol 5-Dimension 5-Level}

The EQ-5D-5L is a self-administered questionnaire in which the respondent records patients' health states in five dimensions: mobility, self-care ability, ability to undertake usual activities, pain and discomfort, and anxiety and depression. Each dimension is scored on five levels: (1) no problems, (2) slight problems, (3) moderate problems, (4) severe problems, and (5) extreme problems. The health states of each hepatitis condition were converted into a weighted health state index by applying scores from the EQ-5D5L preference weights elicited from the general population samples. For this study, Japanese population 
weights were converted to an EQ-5D-5L index score [15].

\section{The utility estimates by hepatitis experts}

A Delphi study was conducted among three experts who engaged in medical care for viral hepatitis over 15 years in a highly advanced medical institution in Japan. First, each of them estimated the utilities of HB- and HC-related diseases, such as AH, FH, ACH, CH, CC, DC, HCC (stage I/II), HCC (stage III/IV), and PLT using the EQ-5D-5L based on a literature review and their experience. Subsequently, a series of panel discussions followed face-to-face at Tokyo University to share each utility estimate and modify the estimates through the Delphi method. The results of $\mathrm{ACH}, \mathrm{CH}, \mathrm{CC}$, and DC were compared to patient-reported scores, and the results of each condition, including more severe ones, were reviewed.

First, each EQ-5 with five response level statements using a five-point Likert scale was assessed individually. Second, additional feedback was provided by three experts. After each round, the results were analyzed and assessed per statement. Consensus was reached when more than $100 \%$ (three experts) of the subjects agreed (strongly agree and agree). When no consensus was reached, the statement was adjusted and presented again in the next round. In these successive rounds, the experts were confronted with deidentified answers from other experts in the previous rounds. After each round, all three experts received a brief summary of the results and were encouraged to give their opinions on the remaining and adjusted statements to reach any consensus on relevant dimensions (Figure 1).

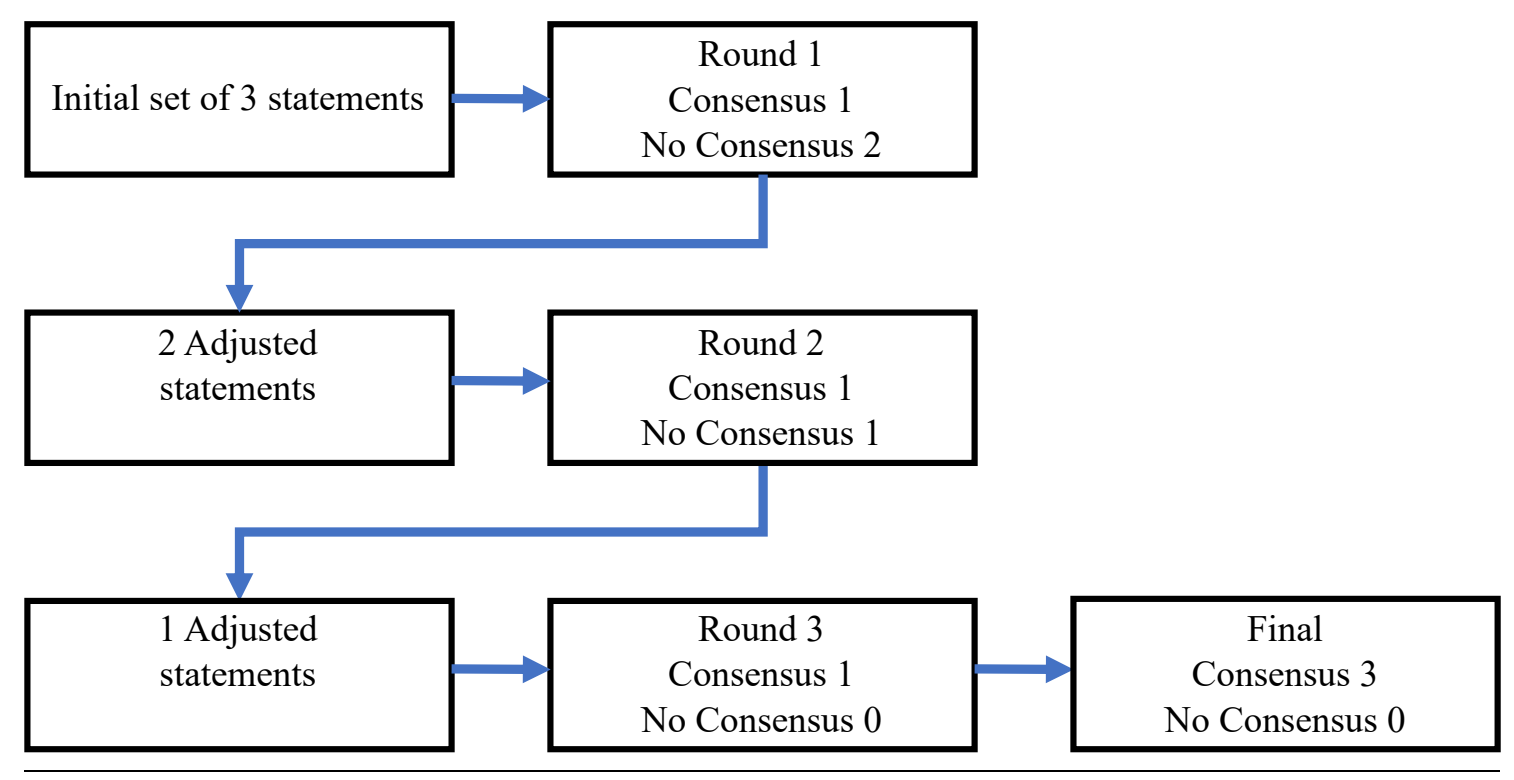

Figure 1. Results per round

\section{Model structure}


Cost-utility models were constructed separately by virus types, incorporating seven phases in chronic $\mathrm{HB}$, two phases in acute $\mathrm{HB}$, and seven phases in chronic $\mathrm{HC}$, and from asymptomatic carriers toward death, based on prior models of the diseases obtained through systematic literature reviews. The models of chronic $\mathrm{HB}$ and $\mathrm{HC}$ are described in Figures 2 and 3, respectively.

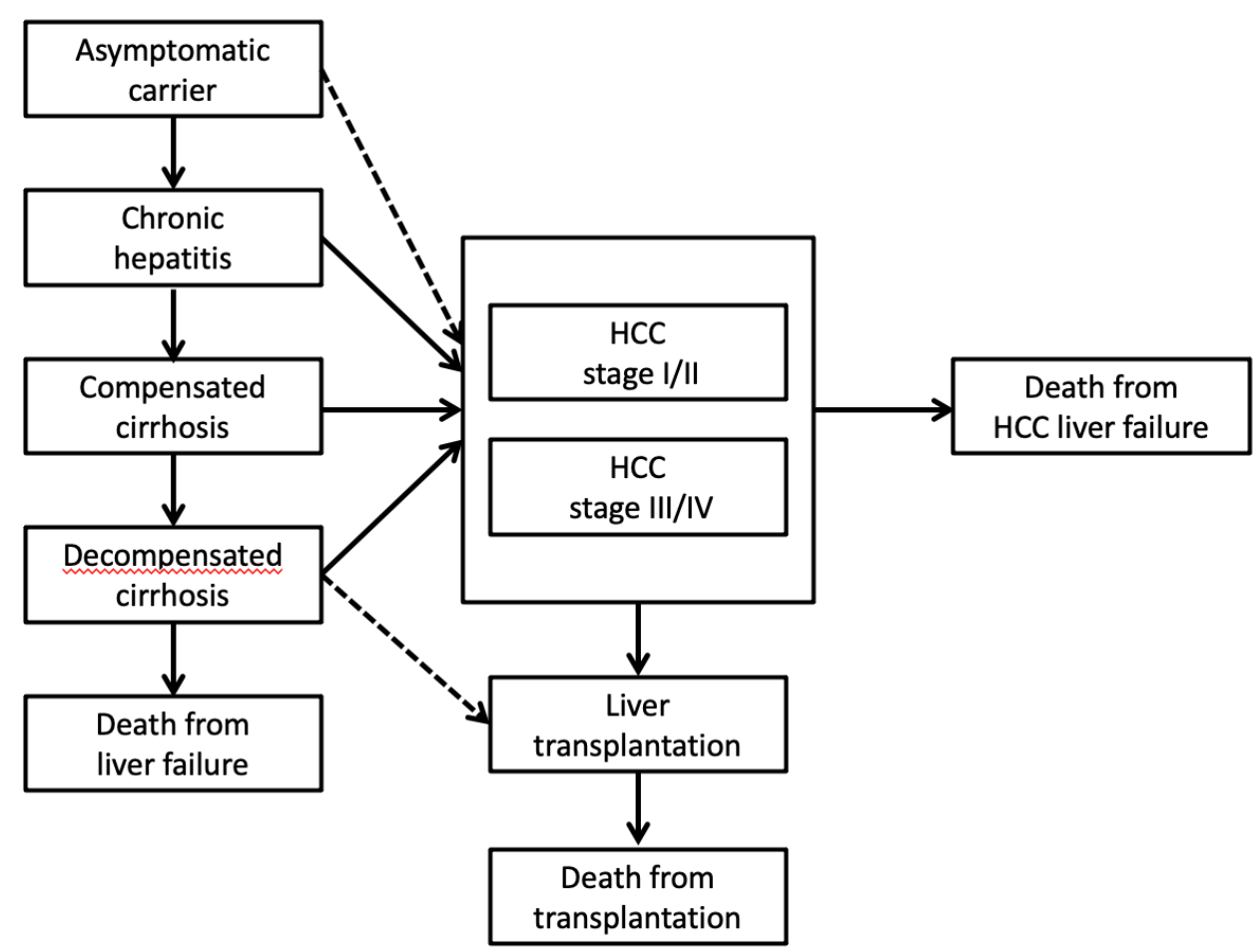

Figure 2. Model structure of chronic hepatitis B-related diseases. 


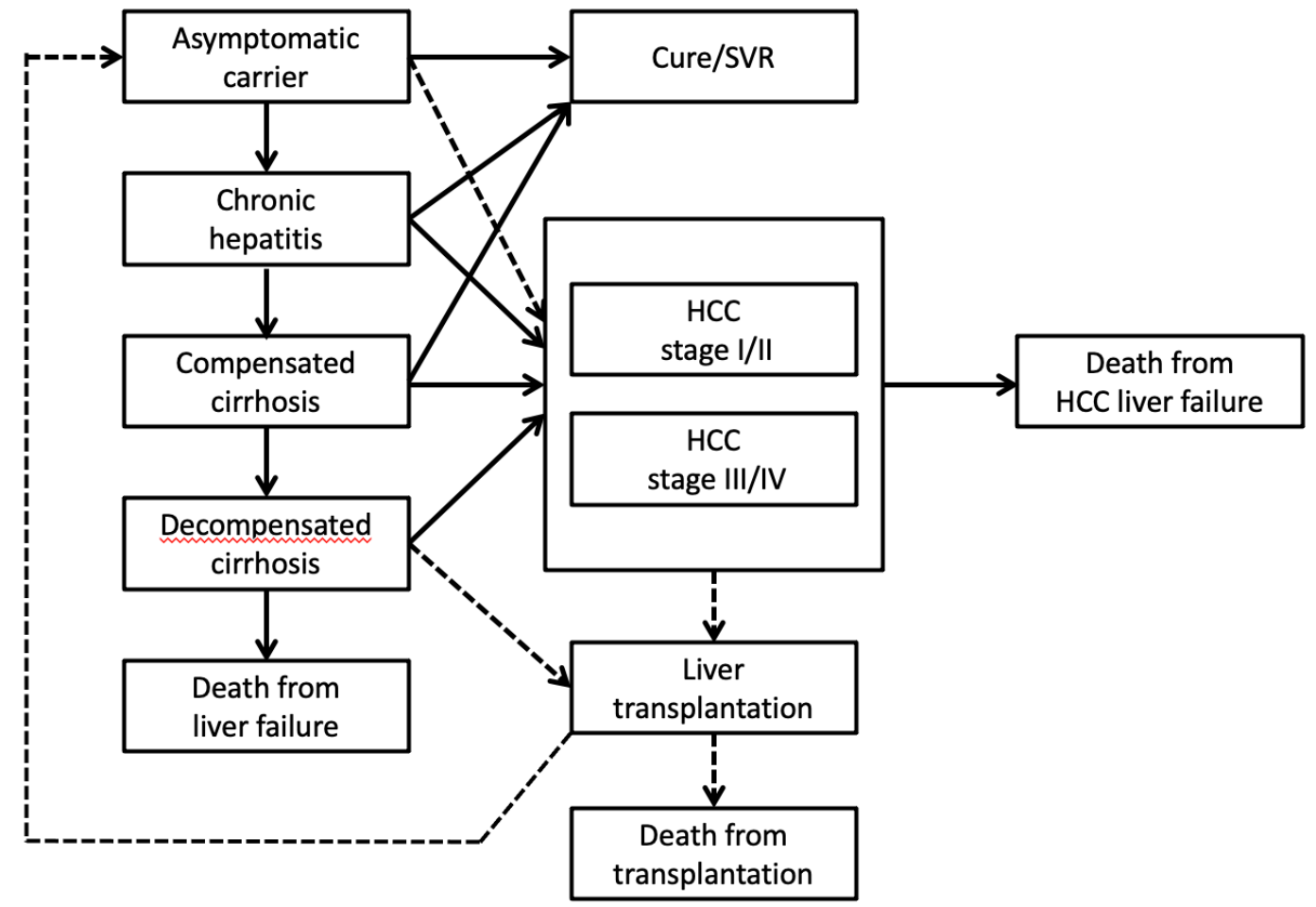

Figure 3. Model structure of chronic hepatitis C-related diseases.

\section{Research ethics approval}

The present study was approved by the National Hospital Organization Nagasaki Medical Center (approval number 23065), and the expert-based study was approved by Kagawa University (approval number Heisei24-099). All methods were carried out in accordance with relevant guidelines and regulations regarding research ethics. Furthermore, informed consent was obtained from all subjects and/or their legal $\operatorname{guardian}(\mathrm{s})$. 


\section{Result}

\section{Patient-reported utilities}

Of the patients $(\mathrm{n}=6331)$ who answered the questionnaire, there were 1197 (622 men and 575 women) eligible subjects and 2508 patients with HC (1039 men and 1469 women). Patient characteristics are shown in Tables 2 and 3.

Table 2. Characteristics of patients with hepatitis $B$.

\begin{tabular}{|l|l|l|l|}
\hline & Male & Female & Total \\
\hline ACH & 120 & 157 & 277 \\
\hline CH & 397 & 347 & 744 \\
\hline CC & 89 & 52 & 141 \\
\hline DC & 16 & 19 & 35 \\
\hline HCC & 99 & 26 & 125 \\
\hline Total & 721 & 601 & 1322 \\
\hline
\end{tabular}

Abbreviations: $\mathrm{ACH}$, asymptomatic chronic hepatitis; $\mathrm{CH}$, chronic hepatitis; $\mathrm{CC}$, compensated cirrhosis; $\mathrm{DC}$, decompensated cirrhosis; HCC, hepatocellular carcinoma

Table 3. Characteristics of patients with hepatitis $\mathrm{C}$.

\begin{tabular}{|l|l|l|l|}
\hline & Male & Female & Total \\
\hline ACH & 80 & 125 & 205 \\
\hline CH & 806 & 1141 & 1947 \\
\hline CC & 110 & 150 & 260 \\
\hline DC & 43 & 53 & 96 \\
\hline HCC & 224 & 143 & 367 \\
\hline Total & 1263 & 1612 & 2875 \\
\hline
\end{tabular}

Abbreviations: $\mathrm{ACH}$, asymptomatic chronic hepatitis; $\mathrm{CH}$, chronic hepatitis; $\mathrm{CC}$, compensated cirrhosis; $\mathrm{DC}$, decompensated cirrhosis; HCC, hepatocellular carcinoma

The utility scores of HB- and HC-related liver diseases, such as ACH, CH, CC, and DC, are shown in Table 4. In patients with chronic $\mathrm{HB}$, the utilities of $\mathrm{ACH}, \mathrm{CH}, \mathrm{CC}$, and $\mathrm{DC}$ were $0.904,0.868,0.845$, and 0.722 , respectively. However, in patients with chronic $\mathrm{HC}$, the utilities under the same conditions were 0.876 , 
$0.821,0.737$, and 0.671 , respectively.

\section{Hepatitis expert-reported utilities}

The utilities of the HB- and HC-related liver diseases, such as AH, FH, HCC, and PLT, were estimated by three hepatitis experts and were averaged out. In patients with acute $\mathrm{HB}$, the utilities of $\mathrm{AH}$ and FH were 0.529 and -0.111 , respectively. In patients with chronic $\mathrm{HB}$ and HC, the utilities of HCC (stage I/II), HCC (stage III/IV), and PLT were 0.675, 0.428, and 0.651, respectively. The utilities reported by the patients and those estimated by the experts are integrated in Table 4.

Table 4. Utilities of hepatitis-related conditions.

\begin{tabular}{|c|c|}
\hline Hepatitis B (acute) & Utility \\
\hline $\mathbf{A H}$ & $0.529 *$ \\
\hline FH & $-0.111 *$ \\
\hline Hepatitis B (chronic) & Utility \\
\hline $\mathbf{A C H}$ & $0.904 * *$ \\
\hline $\mathbf{C H}$ & $0.868 * *$ \\
\hline $\mathbf{C C}$ & $0.845 * *$ \\
\hline DC & $0.722 * *$ \\
\hline HCC (stage I/II) & $0.675^{*}$ \\
\hline HCC (stage III/IV) & $0.428 *$ \\
\hline PLT & $0.651 *$ \\
\hline Hepatitis C & Utility \\
\hline ACH & $0.876 * *$ \\
\hline $\mathbf{C H}$ & $0.821 * *$ \\
\hline $\mathbf{C C}$ & $0.737 * *$ \\
\hline DC & $0.671 * *$ \\
\hline HCC (stage I/II) & $0.675^{*}$ \\
\hline HCC (stage III/IV) & $0.428 *$ \\
\hline PLT & $0.651 *$ \\
\hline
\end{tabular}

*Mean of expert-reported EQ-5D-5L

${ }^{* *}$ Mean of patient-reported EQ-5D-5L

Abbreviations: $\mathrm{AH}$, acute hepatitis; $\mathrm{FH}$, fulminant hepatitis; $\mathrm{ACH}$, asymptomatic chronic hepatitis; $\mathrm{CH}$, chronic hepatitis; CC, compensated cirrhosis; DC, decompensated cirrhosis; HCC, hepatocellular carcinoma; PLT, post-liver transplantation 


\section{Discussion}

This study showed the utilities of both HB- and HC-related diseases in a large-scale Japanese population using the EQ-5D-5L. This study suggests that QOL is markedly diminished in HCB and HCV patients, and the utilities of HB-related diseases were slightly higher than those of HC-related diseases, comparably disease pases. These data are similar to those of previous studies $[10,16]$.

Other studies have reported similar results. For example, Chong et al. [16] have shown that the utilities of moderate HC ( $\mathrm{n}=44)$, HC-related CC $(\mathrm{n}=24)$, DC $(\mathrm{n}=9)$, HCC $(\mathrm{n}=15)$, and PLT $(\mathrm{n}=30)$ in the Canadian population using the EQ-5D-3L were $0.76,0.74,0.66,0.65$, and 0.69 , respectively. These data for DC, HCC, and PLT were similar to those in our study. The patients with HCC in this study were considered to have stage I or II HCC because they were only outpatients. The data for moderate $\mathrm{HC}$ and CC were slightly lower than those in our study. This may be because the utilities in the Canadian population were measured using the EuroQol 5-Dimension 3-Level (EQ-5D-3L), but those in our study were measured using the EQ5D-5L [17]. Moreover, Saeed et al. have recently reported a meta-analysis of health utilities in patients with chronic HC. These utilities were mild/moderate CH (0.751), CC (0.671), HCC (0.662), and DC (0.602) [18]. Their utility data also seem to be slightly lower than those in our study. In a clinical setting involving patients with $\mathrm{CH}$, a relative 7\% reduction in "the ceiling effect" was found in the EQ-5D-5L from that of the EQ-5D-3L, and the EQ-5D-5L was feasible and had promising levels of performance [17].

In contrast, Woo et al. [10] have shown that the utilities of non-cirrhotic HB ( $n=294)$, HB-related CC $(\mathrm{n}=79), \mathrm{DC}(\mathrm{n}=7), \mathrm{HCC}(\mathrm{n}=23)$, and PLT $(\mathrm{n}=30)$ in the Canadian population using the EQ-5D were 0.92, $0.88,0.73,0.81$, and 0.84 , respectively. Moreover, Ong et al. [18] have shown that the utilities of each condition were similar to or slightly higher than Woo's data. In these studies, the utilities of HCC and PLT were higher than those in our study. This may be because the patients in the studies were different from our patients in that their patients were only outpatients and their symptoms might be more moderate.

This study was conducted with reference to the two guidelines of the NICE $[14,20]$. These two guidelines recommend that the EQ-5D is the preferred measure of health-related quality of life in adults. The EQ-5D probably remains the preferred questionnaire on the following grounds: (1) it meets the reference case criteria; (2) it is the most widely used generic preference-based measure; thus, more studies will prefer using this questionnaire; (3) it has been shown to have acceptable psychometric properties across a wide range of common conditions (including rheumatoid arthritis, hip fracture, intermittent claudication, liver transplantation); (4) there is no other generic preference-based measure that is likely to displace this position in the near future; and (5) for the sake of continuity with previous technology appraisals, it is better to use the EQ-5D unless a significantly better generic measure is determined.

The NICE guidelines state that HRQoL, or changes in HRQoL, should be measured directly by patients. Moreover, the utilities estimated by experts are known to be significantly different from those reported by patients $[16,22,23]$. It has been reported that quality of life indices tend to be overly valued by 
clinicians [22]. However, some authors have described that the proxy could assess the patient's mobility and self-care accurately [24] and moderate agreement between responses from patients and those from their proxies for at least the observable domains of the EQ-5D [24, 25]. In general, some domains of EQ-5D obtained from a proxy may be sufficiently valid and unbiased for use in most types of trials and surveys [24]. In another study [26], the total EQ-5D score was significantly positively correlated between patients at the end stage of lung cancer reporting and nurses reporting. Moreover, the scores for some items, including "mobility," "self-care," and "pain/discomfort" of EQ-5D, have statistically significant positive correlation between patients and nurses [26]. In terms of the Chronic Liver Disease Questionnaire (CLDQ), a liver disease-specific instrument used for the assessment of HRQoL, since the HRQOL of patients with HCC is greatly altered both qualitatively and quantitatively, they were not included in the study that developed CLDQ [26,27].

In this study, data from more severe conditions were estimated by three hepatitis experts. Because our study mainly comprised outpatients, data of patients with severe hepatitis who were largely admitted to hospitals were biased, and these patients may have difficulty in evaluating their own conditions [25]. Certainly, the utility of HCC reported by outpatients in our study was relatively higher than that reported in other studies $[16,28]$, because their symptoms were relatively moderate. Subsequently, the utilities of more severe hepatitis-related diseases, such as $\mathrm{HCC}, \mathrm{AH}, \mathrm{FH}$, and PLT, were estimated by hepatitis experts. Moreover, since there is the potential for patients not to recognize their own conditions correctly, in a study based on a self-administered questionnaire, disease conditions might be misclassified. Furthermore, hepatitis expert-reported utilities in less severe conditions, such as $\mathrm{ACH}, \mathrm{CH}, \mathrm{CC}$, and $\mathrm{DC}$, were similar to those of patient-reported utilities under the same conditions. This might suggest that our results from hepatitis expert-reported utilities in severe conditions such as HCC (stage I/II), HCC (stage III/IV), PLT, $\mathrm{AH}$, and $\mathrm{FH}$ were reasonable and reliable. However, the value judgment of the utilities requires further investigation. 


\section{Acknowledgment}

This study was supported in part by the Health and Labour Sciences Research Grants (\#14428223, "Medical economic assessment for viral hepatitis strategy in Japan”, 2016) and the Health and Labour Sciences Research Grants (\#1901FB01, "Study of intervention effects of health-checkups for unique female's diseases ", 2019) from the Ministry of Health, Labour and Welfare of Japan, and the Grant-in-Aid for Scientific Research (C) (\#20298026, “Health literacy and QOL(EQ-5D-Y) in Japanese children”, 2020) from the Ministry of Education, Culture, Sports, Science and Technology (MEXT) of Japan.

\section{Disclosure of interest}

The authors have no conflicts of interest directly relevant to the content of this article.

\section{References}

1. Di Bisceglie AM. Hepatitis B and hepatocellular carcinoma. Hepatology. 2009;49:S56-S60.

2. Di Bisceglie AM. Hepatitis C and hepatocellular carcinoma. Semin Liver Dis. 1995;15:64-69.

3. Parkin DM. Global cancer statistics in the year 2000. Lancet Oncol. 2001;2:533-543.

4. Higuchi M., Tanaka E. \& Kiyosawa K. Epidemiology and clinical aspects on hepatitis C. Jpn J Infect Dis. 2002;55:69-77.

5. Ishida H., Inoue Y., Wong JB. \& Okita K. Cost-effectiveness of ribavirin plus interferon alpha-2b for either interferon relapsers or non-responders in chronic hepatitis C: a Japanese trial. Hepatol Res. 2004;28:125-136.

6. Chung H., Ueda T. \& Kudo M. Changing trends in hepatitis C infection over the past 50 years in Japan. Intervirology. 2010;53:39-43.

7. Iwasawa K. et al. Hepatitis B (HB) immunoglobulin plus $\mathrm{HB}$ vaccine for intrauterine HB virus infection. Pediatr Int. 2015;57:401-405.

8. Mizokami M. et al. Ledipasvir and sofosbuvir fixed-dose combination with and without ribavirin for 12 weeks in treatment-naive and previously treated Japanese patients with genotype 1 hepatitis C: an open-label, randomised, phase 3 trial. Lancet Infect Dis. 2015;15:645-653.

9. Li HC. \& Lo SY. Hepatitis C virus: virology, diagnosis and treatment. World J Hepatol. 2015;7:1377-1389.

10. Woo G. et al. Health state utilities and quality of life in patients with hepatitis B. Can J Gastroenterol. 2012;26:445-451.

11. McLernon DJ., Dillon J. \& Donnan PT. Health-state utilities in liver disease: a systematic review. Med Decis Making. 2008;28:582-592. 
12. Badia X., Roset M., Herdman M. \& Kind P. A comparison of United Kingdom and Spanish general population time trade-off values for EQ-5D health states. Med Decis Making. 2001;21:7-16.

13. Weinstein MC., Torrance G. \& McGuire A. QALYs: the basics. Value Health. 2009;12:S5-S9.

14. National Institute for Health and Care Excellence. Guide to the methods of technology appraisal 2013. 2013.

15. Ikeda S. et al. Developing a Japanese version of the EQ-5D-5L value set. J Natl Inst Public Health. $2015 ; 64: 47-55$.

16. Chong CA. et al. Health-state utilities and quality of life in hepatitis C patients. Am J Gastroenterol. 2003;98:630-638.

17. Scalone L. et al. Comparing the performance of the standard EQ-5D 3L with the new version EQ5D-5L in patients with chronic hepatic diseases. Qual Life Res. 2013;22:1707-1716.

18. Saeed YA. et al. A systematic review and meta-analysis of health utilities in patients with chronic hepatitis C. Value Health. 2020;23:127-137.

19. Ong SC, Mak B, Aung MO, Li SC \& Lim SG. Health-related quality of Life in chronic hepatitis B patients. Hepatology.2008; 47:1108-1117.

20. National Institute for Health and Clinical Excellence. Briefing paper for methods review workshop on key issues in utility. 2007.

21. Younossi ZM, Boparai N, McCormick M, Price LL \& Guyatt G. Assessment of utilities and healthrelated quality of life in patients with chronic liver disease. Am J Gastroenterol. 2001;96:579-583.

22. Wells CD, Murrill WB \& Arguedas MR. Comparison of health-related quality of life preferences between physicians and cirrhotic patients: implications for cost-utility analyses in chronic liver disease. Dig Dis Sci. 2004;49:453-458.

23. Meyers AR, Gage H \& Hendricks A. Health-related quality of life in neurology. Arch Neurol. 2000;57:1224-1227.

24. Dorman PJ, Waddell F, Slattery J, Dennis M \& Sandercock P. Are proxy assessments of health status after stroke with the EuroQol questionnaire feasible, accurate, and unbiased? Stroke. 1997;28:18831887.

25. Magaziner J, Zimmerman SI, Gruber-Baldini AL, Hebel JR \& Fox KM. Proxy reporting in five areas of functional status. Comparison with self-reports and observations of performance. Am J Epidemiol. 1997;146:418-428.

26. Takura T, Koike T, Sekimoto A \& Mutou M. Agreement between patient's and proxies reports of health related quality of life in patients with end-stage lung cancer in Japan. J Nurs Care. 2014;3:7.

27. Younossi ZM, Guyatt G, Kiwi M, Boparai N \& King D. Development of a disease specific questionnaire to measure health related quality of life in patients with chronic liver disease. Gut. 1999;45:295-300.

28. Tanaka A. et al. Validation of the Japanese version of the Chronic Liver Disease Questionnaire for 
the assessment of health-related quality of life in patients with chronic viral hepatitis. Hepatol Res. 2016;46:E45-E50.

29. John-Baptiste AA. et al. Sustained responders have better quality of life and productivity compared with treatment failures long after antiviral therapy for hepatitis C. Am J Gastroenterol. 2009;104:2439-2448. 